\title{
UPDATE ON THE POWERING STRATEGY OF LHC
}

\author{
P. Proudlock, on behalf of the LHC Electrical Engineering and Dynamic Effects Working Groups, \\ CERN, 1211 Geneva 23
}

\begin{abstract}
Over the last two years the powering strategy of the Large Hadron Collider (LHC) has undergone a considerable evolution. This was not only motivated by new requirements but more importantly by a quest to reduce costs and improve operational reliability and flexibility. In order to achieve this, despite an increase in complexity, novel modifications to the strategy and hardware have been proposed both on the warm and cold sides of the machine. During this process the requirements of the accelerator physics have been clarified and met while working within the boundary conditions imposed by the utilisation of the existing CERN infrastructure. After a brief review of the present powering strategy which needs to produce and supply 2MA dc in 1800 circuits, more detailed descriptions will be given of the changes implemented in order to achieve a more rational overall system. Some performance issues of the powering system will also be discussed.
\end{abstract}

\section{INTRODUCTION}

The LHC will be installed in an existing underground tunnel of $27 \mathrm{~km}$ circumference that presently houses the Large Electron Positron machine (LEP). Much of the infrastructure of LEP will be reused, notably the civil engineering and electrical distribution which, while giving substantial economies, will place constraints on the powering equipment which must be designed within certain boundary conditions.

Each of the eight sectors of LHC will be powered individually and, for the main circuits, ring 1 and 2 will be powered in series. However, the main dipoles, focusing quadrupoles and defocusing quadrupoles of the FODO cell structure are separately powered. A continuous cryostat runs along each of the eight sectors with superconducting magnets cooled to < $2 \mathrm{~K}$ (Fig. 1). This cryostat contains the main lattice circuits as well as the dispersion suppressors and some of the matching section at each end. A large number of superconducting bus-bars run through the cryostats connecting the various circuits which are fed from electrical feed boxes (DFB) located at each end of the sectors. The long straight sections of the interaction regions have several additional magnets that are locally fed. The main lattice circuits are fed from the even points, left and right, while in the odd points the powering is kept to a minimum and is associated with the circuits located at the end of the arcs.
This matches the geographic availability of the existing ac power of LEP.

The power converters of LHC must be situated as close as possible to the machine's electrical feed boxes in order to minimize the transport of the high currents required by the machine (up to $13 \mathrm{kA}$ ). Therefore, they will be placed underground using, for the most part, the caverns vacated by the Klystrons of LEP (Fig. 2). The LHC has the following superconducting circuits;

- 24 main circuits of $13 \mathrm{kA}$,

- 8 low beta triplets requiring 8 or $13 \mathrm{kA}$,

- 212 individually powered quadrupole circuits requiring $6 \mathrm{kA}$,

- 544 auxiliary circuits of $600 \mathrm{~A}$,

- 956 orbit correctors of 60 to $120 \mathrm{~A}$.

There are also 48 circuits with room temperature magnets.

In all, LHC will require $2000 \mathrm{kA}$ through 1800 circuits.

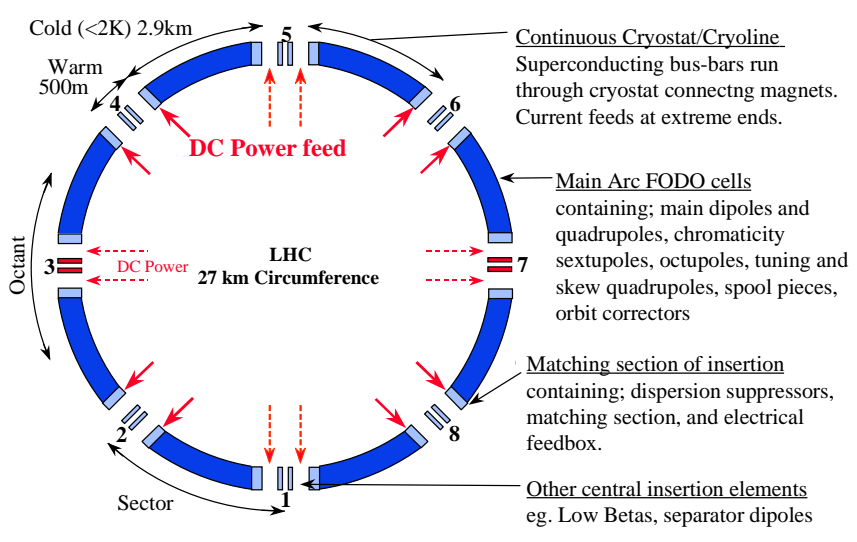

Figure 1: Schematic layout of LHC.

\section{MAIN DIPOLES AND THEIR CORRECTORS}

Each of the eight main dipole circuits contains 154 dipoles. They have cold bypass diodes and energy extraction to deal with a quench in one or more of the magnets [1]. Half the magnets are placed on the go busbar and half on the return so that energy extraction can be performed at the two ends of a sector, thus reducing the voltage withstand requirements. This results in two types of main dipole, at least as far as the connections are concerned. Originally b3 and b5 correctors were placed in all dipole magnets and later the possibility of adding a4 


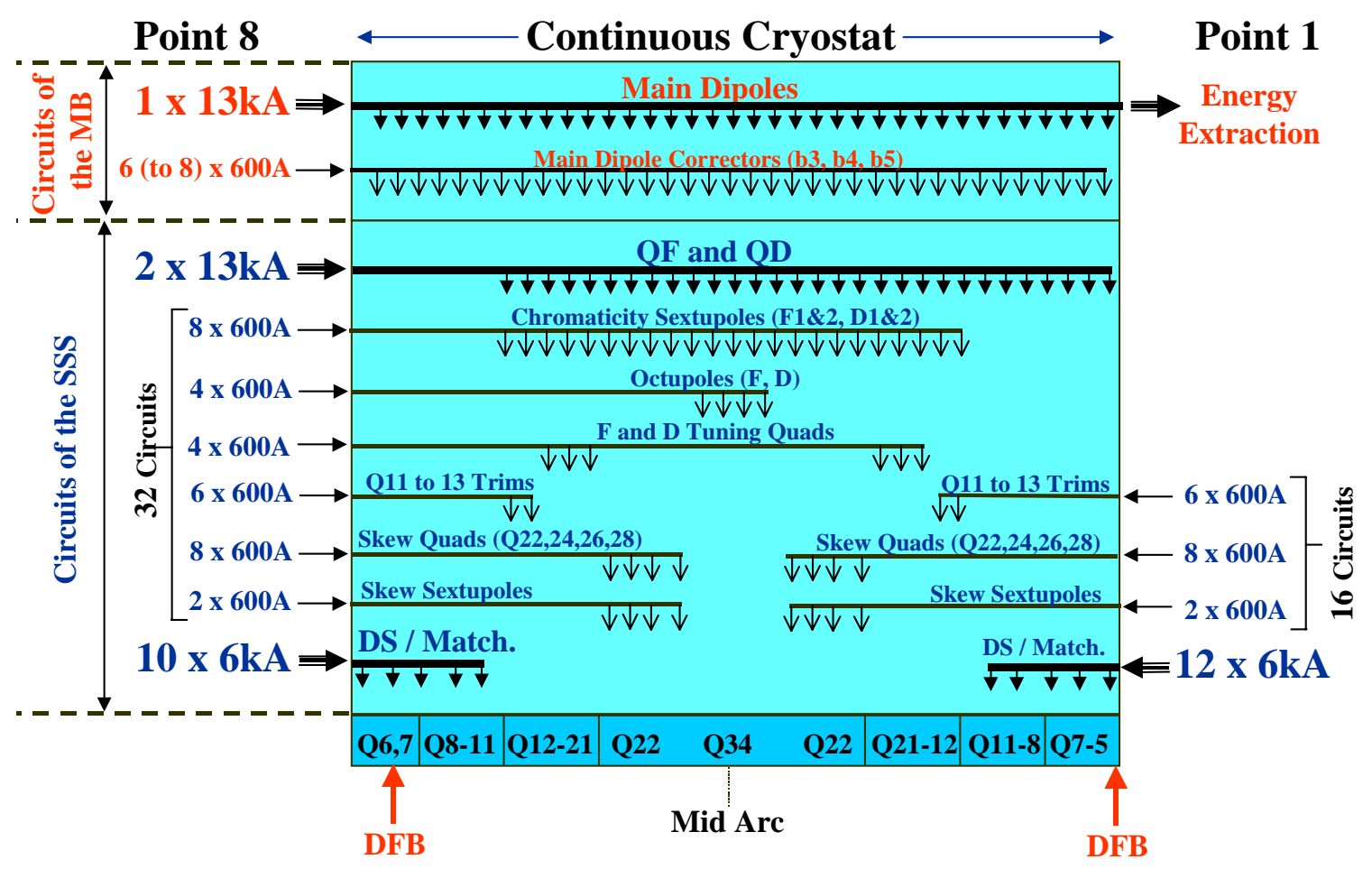

Figure 2: Schematic layout of sector of LHC.

and b4 correctors was considered. However, the exact requirements will not be known until the first pre-series magnets are measured. It has therefore been decided to profit from the existence of two types of dipole. One type will be equipped with a full complement of correctors (nested $\mathrm{b} 4 / \mathrm{b} 5$ on one side and $\mathrm{b} 3$ on the other) while the other will have only a b3 corrector thus leaving space available if extra correctors are needed following measurements on the first series magnets. Provision for powering these additional circuits will be made.

\section{INSERTION QUADRUPOLES}

Originally the main arc quadruples were used for the individually powered quadrupoles of the long straight sections. These required $13 \mathrm{kA}$ and, while feasible to power in a chain, were very expensive to power individually. A new magnet has now been designed specifically for such applications, which requires a maximum of $6 \mathrm{kA}$. Later studies on the dispersion suppressor revealed problems to get an adequate range from the trim system [2]. Therefore, these same magnets are now used in the dispersion suppressor and can be individually powered. Three rather than four bus-bars are used to power the two apertures thus saving on cold busbars and space requirements. The trim system is however retained for the dispersion suppressors of the cleaning insertions, but they no longer require the longer version of the main quadrupole. Thus only one length of main quadrupole is now necessary.

\section{SUPERCONDUCTING BUS-BARS}

The circuits associated with the main dipoles (dipole plus corrector magnets) as well as the two quadrupole circuits are fed through the cold masses of all magnets (Fig. 3). Their bus-bars are installed in slots in the cold masses. The dipoles use two bus-bars of $7.4 \mathrm{~mm}^{2} \mathrm{NbTi}$ with $300 \mathrm{~mm}^{2}$ of copper stabiliser. Energy is extracted, in case of a quench, at an initial rate of $125 \mathrm{~A} / \mathrm{s}$. The quadrupoles use four bus-bars of $7.4 \mathrm{~mm}^{2} \mathrm{NbTi}$ with $180 \mathrm{~mm}^{2}$ of copper and the initial discharge rate is $320 \mathrm{~A} / \mathrm{s}$. The dipole corrector magnets use 20 monolithic rectangular conductors with a cross section of $3 \mathrm{~mm}^{2}$ and a copper to superconducting ratio of 9:1.

The individually powered quadrupoles and the array of corrector circuits associated with the short straight section (SSS) need to bypass the dipoles and a link is provided along the 53 metres of the half cell. For the 600 A circuits a flexible multi-wire cable is used containing 36 superconducting wires with a diameter of $1.6 \mathrm{~mm}$ and a ratio $\mathrm{Cu} / \mathrm{SC}$ ratio of 9.5:1. The outer diameter of the complete cable is $15.5 \mathrm{~mm}$. A similar cable is proposed for the $6 \mathrm{kA}$ circuits containing 18 multifilament conductors and having an external diameter of $23 \mathrm{~mm}$. These need to be installed in a tube containing helium II running from SSS to SSS [3]. The number and type of cable varies around the machine according to the local needs for powering. This solution, compared to previous designs, where all routing was done inside the cold masses, reduces the number of electrical interconnections by 48000 and yields 


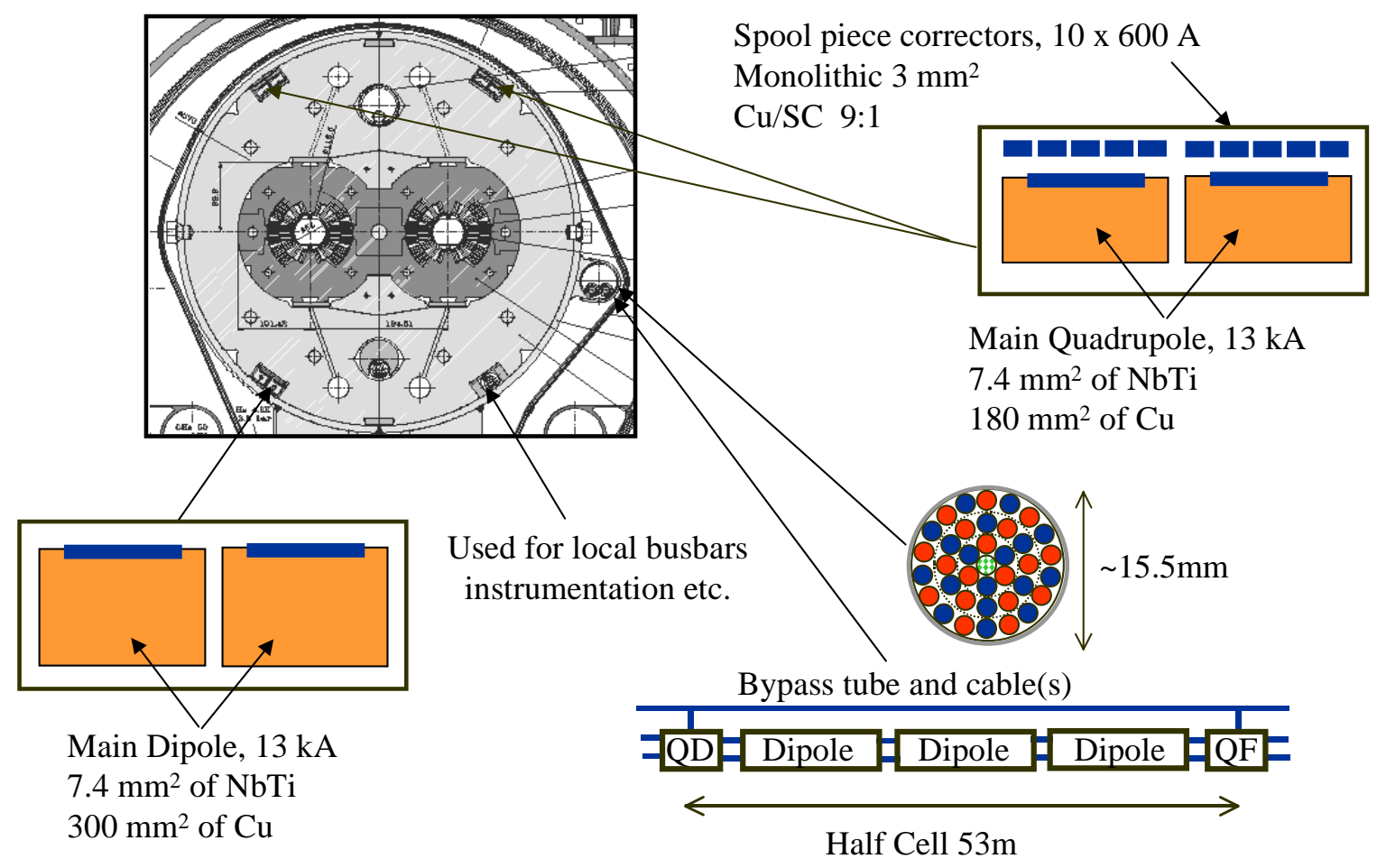

Figure 3: Superconducting busbars.

flexibility and reduction in costs. The $6 \mathrm{kA}$ cable is the most difficult to engineer since the forces acting on it will be appreciable as there is a non-negligible field in the region of the tube. The tube itself needs to be carefully thermalised to the cold mass in order to avoid unacceptable additional cool down times.

\section{POWER CONVERTER PERFORMANCE}

It has now been established that modular switch-mode power converters with digital current loops will be used for LHC [4]. Some circuits will need a performance of a few parts per million of maximum and resolution of 20 bits. The reference magnets, previously in series with each of the main lattice circuits ( 24 in all), have now been eliminated. A few remote reference magnets powered by the same current cycle as those in the machine will replace them. They will be used primarily to derive feedback for the dipole correction circuits during persistent current decay and snap-back. Work has continued on beam feedback for orbit, tune and chromaticity [5]. An overall bandwidth of $1 \mathrm{~Hz}$ will be necessary and deterministic control will be provided using commercially available field busses

\section{CONCLUSIONS}

Over the last two years the powering strategy of LHC has undergone a considerable evolution. This was not only motivated by new requirements but more importantly by a quest to reduce costs and improve operational reliability and flexibility. During this process the requirements of the accelerator physics have been clarified and met while working within the boundary conditions imposed by the utilisation of the existing CERN infrastructure.

\section{ACKNOWLEDGEMENTS}

The members of the Electrical Engineering and Dynamic Effects working groups of the LHC Project carried out much of the work reported above and thanks go to them.

\section{REFERENCES}

[1] F. Rodriguez-Mateos, The Protection System for the Superconducting Elements of the LHC, this conference.

[2] P. Proudlock, Electrical Powering Strategy of LHC, First design study, P. Proudlock, $5^{\text {th }}$ EPAC, 10-14 June 1996, Sitges, Spain.

[3] A. Poncet, Update of the LHC Arc System Layouts and Integration, this conference.

[4] F. Bordry et al, Four-Quadrant Converter (600 A, 12 V) - LHC Converter Prototype, this conference.

[5] R. Bailey et al., Dynamic Effects and their Control at the LHC, PAC, 12-16 May 1997, Vancoucer, Canada. 